\title{
Finite Element Analysis of the Nanomechanics of Hard Coatings on a Soft Polymer Substrate by a Spherical Indenter
}

\author{
Chunlai Tian ${ }^{1}$ and Pengfei Duan $\mathbb{D}^{2}$ \\ ${ }^{1}$ School of Mechanical and Electronic Engineering, Pingxiang University, Pingxiang, Jiangxi, China 337000 \\ ${ }^{2}$ Beijing Exchange Center of Science and Technology, Beijing Academy of Science and Technology, Beijing, China 100089
}

Correspondence should be addressed to Pengfei Duan; duanpengfei@becst.net.cn

Received 14 February 2020; Revised 5 April 2020; Accepted 8 April 2020; Published 24 April 2020

Academic Editor: Gyorgy Szekely

Copyright (c) 2020 Chunlai Tian and Pengfei Duan. This is an open access article distributed under the Creative Commons Attribution License, which permits unrestricted use, distribution, and reproduction in any medium, provided the original work is properly cited.

\begin{abstract}
Composite has been widely used in various fields due to its advanced performance. To reveal the relation between the mechanical properties of the composite and that of each individual component, finite element analysis (FEA) has usually been adopted. In this study, in order to predict the mechanical properties of hard coating on a soft polymer, the response of this coating system during nanoindentation was modelled. Various models, such as a viscoelastic model and fitting model, were adopted to analyse the indentation response of this coating system. By varying the substrate properties (i.e., Young's modulus, viscoelasticity, and Poisson's ratio), Young's modulus, energy loss, and the viscoelastic model of the coating system were analysed, and how the mechanical properties of the substrate will affect the indentation response of the coating system was discussed.
\end{abstract}

\section{Introduction}

Recently, coating is becoming increasingly important. Various functional coatings have been adopted to improve the surface properties of the substrate, such as oxide coating which has been applied to polymer substrates and ceramic coating which has been applied to metal substrates [1-6]. The mechanical properties of these coatings are important in determining their lifetime. However, conventional techniques are incapable of measuring the mechanical properties of thin coating layers. Therefore, nanoindentation has been adopted, which is an efficient technique for determining mechanical properties of thin coatings. This measurement has the features of operating at nano- to submicroscale and recording both indentation force and penetration, from which mechanical properties of the specimen (such as Young's modulus, hardness, and stress relaxation) can be deconvoluted [7-9].

This test has been extensively studied in recent years; increasing attention has been transferred from measuring the mechanical properties of single material to determining the mechanical properties of a coating/substrate system, as the latter one is always economically relative. In a coating/substrate system, with the increase of the indentation depth, the substrate deformation starts playing an important role in the indentation response of the coating [7]. Therefore, this study investigates how the substrate deformation may affect the indentation response of the coating during a nanoindentation test.

\section{Methodology}

2.1. Finite Element Model. The substrate deformation consists of elastic and plastic deformation, and the deformation during indentation is much more complicated. Hence, the finite element analysis has become a major technique to simulate the response of hard coating on a soft polymer indented by a spherical tip. This study investigates the influences of the substrate on the nanoindentation response, by changing the parameters of the polymer, such as Young's modulus and viscoelasticity. 


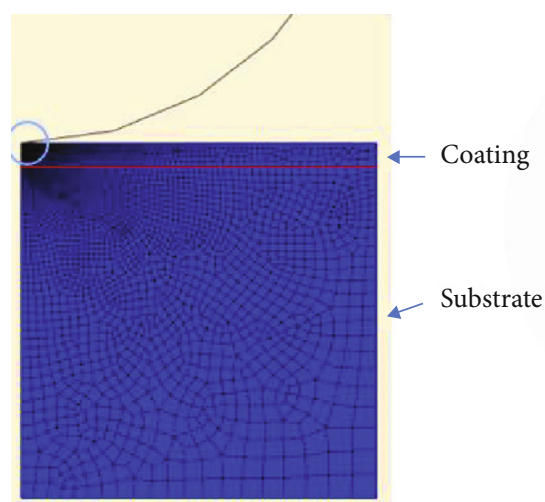

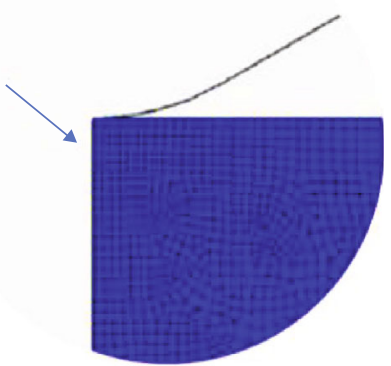

(b)

(a)

FIGURE 1: Finite element mesh for a spherical tip indenting hard coating on a soft substrate. (a) The overview of the coating/substrate system. (b) Enlarged details of coating elements underneath the spherical tip.

The classical Hertz contact model has been used to determine $E_{t=0}$ or $E_{\infty}$. For nanoindentation with a loading-holding protocol, the relationship between force and displacement for a ramping period is given by [10]

$$
P=\frac{4 E R^{1 / 2}}{3\left(1-v^{2}\right)} \delta^{3 / 2}
$$

where $P$ is the force, $\delta$ is the displacement, $E$ is Young's modulus, $R$ is the effective radius, and $v$ is Poisson's ratio.

During the stress relaxation period, the force-displacement relation is given by [10]

$$
P=\frac{4 E R^{1 / 2}}{3\left(1-v^{2}\right)} \delta^{3 / 2}\left(1+k \exp \left(-\frac{\mathrm{t}}{\tau_{r}}\right)\right),
$$

where $t$ is time, $k$ is the material-related constant, and $\tau_{r}$ is the time constant.

ABAQUS 6.10 has been used as the finite element analysis software. Due to the symmetric nature of the indenter and coating/substrate system, a 2D axisymmetric model was built up instead of a 3D model. In which case, the elastic-plastic deformation responses from those two models are identical [11], while the former model gives more computational efficiency. Figure 1 shows the details of finite element meshes: (a) an overview of the coating/substrate system and (b) enlarged detail of coating elements underneath the tip. The element type is CAX4R, which is a 4-node bilinear axisymmetric quadrilateral with reduced integration. A total of 9822 elements were adopted to model the coating/substrate system. Finer elements were arranged underneath the indenter to provide more accurate simulation results. The interface between the coating and the substrate can be as complicated as dynamically bonded, such as biological cells for active materials [12-14]. In this study, the emphasis is on the mechanical properties of hard coating on a soft polymer. Thus, it was assumed to be perfectly bonded here. A completely fixed boundary condition was applied to the bottom of the composite model. An axisymmetric boundary condition was applied to the symmetry axis of the indenter and the system.
In the model, a spherical tip with a radius of $150 \mu \mathrm{m}$ was used. Glass was adopted as the hard coating, in which thickness was fixed to $10 \mu \mathrm{m}$. This glass has Young's modulus of $70 \mathrm{GPa}$. The substrate used polymer as its material, in which Young's modulus was changed from $0.7 \mathrm{GPa}$ to $7 \mathrm{GPa}$. In order to obtain more accurate results, Poisson's ratios of both glass and polymer were fixed to 0.3 at the beginning, after which $v$ (glass) changed to 0.25 and $v$ (polymer) changed to 0.5 to tell the influences from Poisson's ratio. For viscoelastic materials, an inappropriate loading rate will significantly affect the mechanical response. It may result in underestimated contact stiffness or even negative contact stiffness during a loading-unloading nanoindentation test. To minimize such effect, a holding period at the maximum load was adopted. As depicted in Figure 2, a maximum penetration of $5 \mu \mathrm{m}$ was adopted with a ramping-holding procedure (ramping period is $1 \mathrm{~s}$, holding period is $10 \mathrm{~s}$ ). To minimize the efforts of friction and boundaries on the simulations, it was assumed that the contact between the indenter and the sample surface was assumed to be frictionless, the width and height of the coating/substrate system were sufficiently large compared to the indentation depth, and materials were isotropic and homogeneous.

2.2. Viscoelastic Model. For viscoelastic materials such as cells, the Hertz contact model cannot be directly adopted, which may lead to an overestimation of Young's modulus. A viscoelastic model is usually used to investigate Young's modulus of the material $[15,16]$. In the simulations, the Prony series model was used to describe the viscoelastic behaviour of the coating/substrate system. The normalized relaxation modulus $(g(t))$ is given by $[10,17,18]$

$$
\begin{aligned}
g(t) & =g_{\infty}+\sum g_{i} \exp \left(-\frac{t}{\tau_{i}}\right), \\
g_{\infty}+\sum g_{i} & =1,
\end{aligned}
$$

where $g_{\infty}$ is the normalized equilibrium modulus, $g_{i}$ is the material-related constant, $t$ is time, and $\tau_{i}$ is the time 


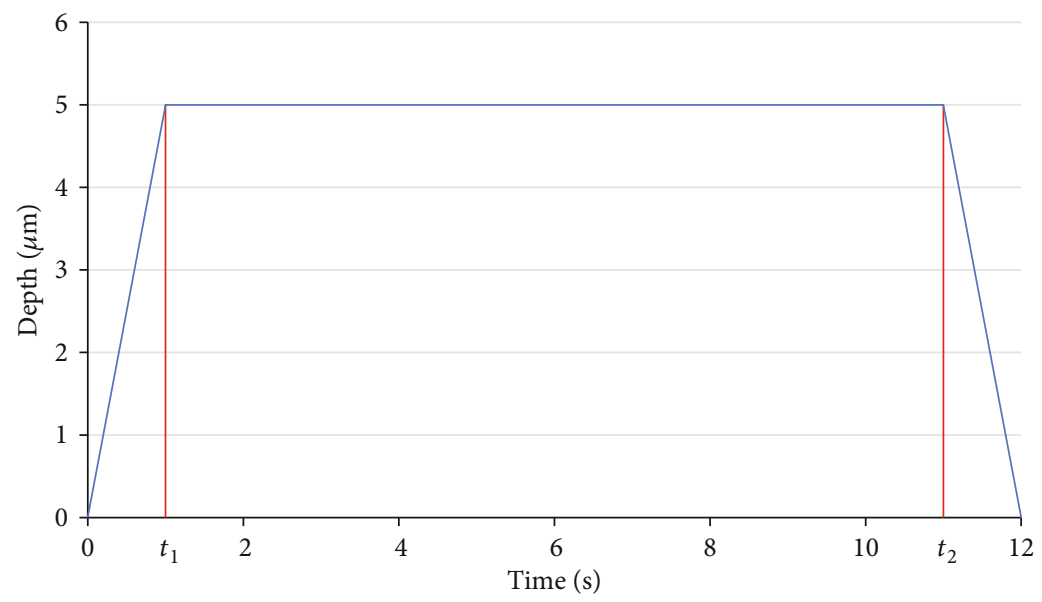

FIGURE 2: Ramping-holding-unloading procedure.

constant. In this study, $\tau_{i}$ is fixed to $0.1 \mathrm{~s}$, and $i$ is assumed to be 1 . Therefore, Young's modulus is given by

$$
E=E_{t=0} g_{\infty},
$$

where $E_{t=0}$ is the instantaneous elastic modulus.

Hence, the force-displacement relations shown in Equations (1) and (2) can be rearranged based on time. During the stress relaxation period, $t_{1} \leq t \leq t_{2}$, Equation (2) can be rewritten as

$$
\begin{aligned}
P(t)= & \frac{4}{3\left(1-v^{2}\right)} \sqrt{R} \delta^{3 / 2}(t) E_{t=0}\left[g_{\infty}\right. \\
& \left.+g_{1} \frac{\tau}{t_{1}} \exp \left(\frac{-t}{\tau}\right)\left[\exp \left(\frac{t_{1}}{\tau}\right)-1\right]\right] .
\end{aligned}
$$

While during the loading period, when $0 \leq t \leq t_{1}$, Equation (1) can be correspondingly rewritten as

$$
\begin{aligned}
P(t)= & \frac{4}{3\left(1-v^{2}\right)} \sqrt{R} \delta^{3 / 2}(t) E_{t=0}\left[g_{\infty}\right. \\
& \left.+g_{1} \frac{\tau}{t}\left(1-\exp \left(-\frac{t}{\tau}\right)\right)\right] .
\end{aligned}
$$

2.3. Fitting Module. After obtaining the data of $E$ (composite) and the relative radius of contact, $x$, an equation proposed by Clifford and Seah was used to fit the relationship between them, which is given by [19-21]

$$
\frac{E^{*}-E_{\mathrm{s}}^{*}}{E_{\mathrm{c}}^{*}-E_{\mathrm{s}}^{*}}=\frac{P x^{n}}{1+P x^{n}+Q x},
$$

where

$$
\begin{aligned}
x & =\frac{\sqrt{R \delta}}{h_{\text {thickness }}}, \\
E^{*} & =\frac{E}{1-v^{2}} .
\end{aligned}
$$

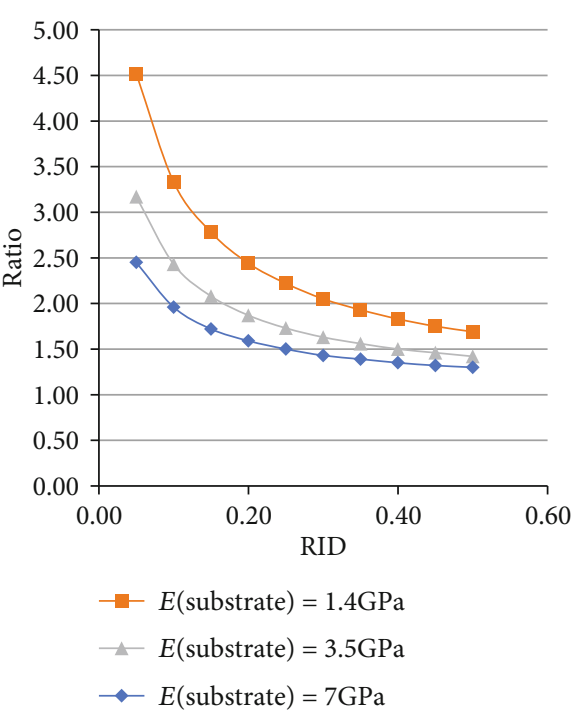

Figure 3: Ratios of Young's modulus based on Equation (12) as a function of RID.

Commercial software MATLAB has been adopted to perform the curve fitting procedure, by using the least mean square approach. In fact, it was found that the results from Equation (7) showed a high level of uncertainty, as $Q$ changed in a certain range. To avoid this uncertainty, Equation (7) was modified as

$$
\frac{E^{*}-E_{\mathrm{s}}^{*}}{E_{\mathrm{c}}^{*}-E_{\mathrm{s}}^{*}}=\frac{P x^{n}}{1+P x^{n}+Q x}+1 .
$$

Compared to Equation (7), this one gives the advantages of high stability and meeting the initial boundary condition (i.e., when $x$ is approaching to $0,\left(E^{*}-E_{\mathrm{s}}^{*}\right) /\left(E_{\mathrm{c}}^{*}-E_{\mathrm{s}}^{*}\right)$ should be 0$)$. This reflects the physical insight that, when the indentation depth is sufficiently small, only the elastic modulus of the coating will be measured. 


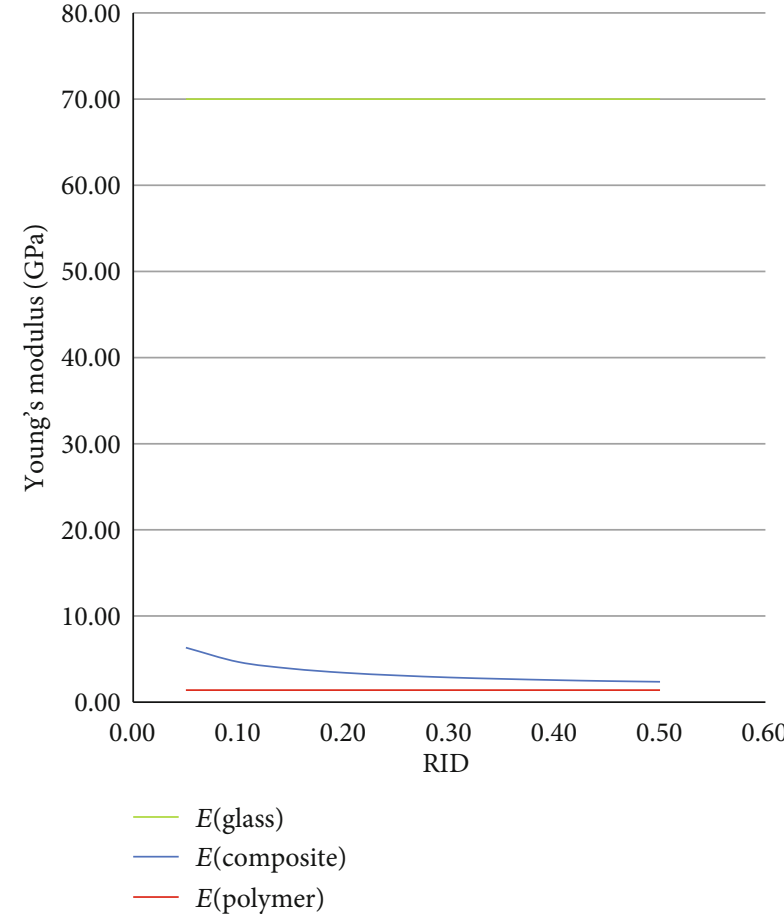

(a)

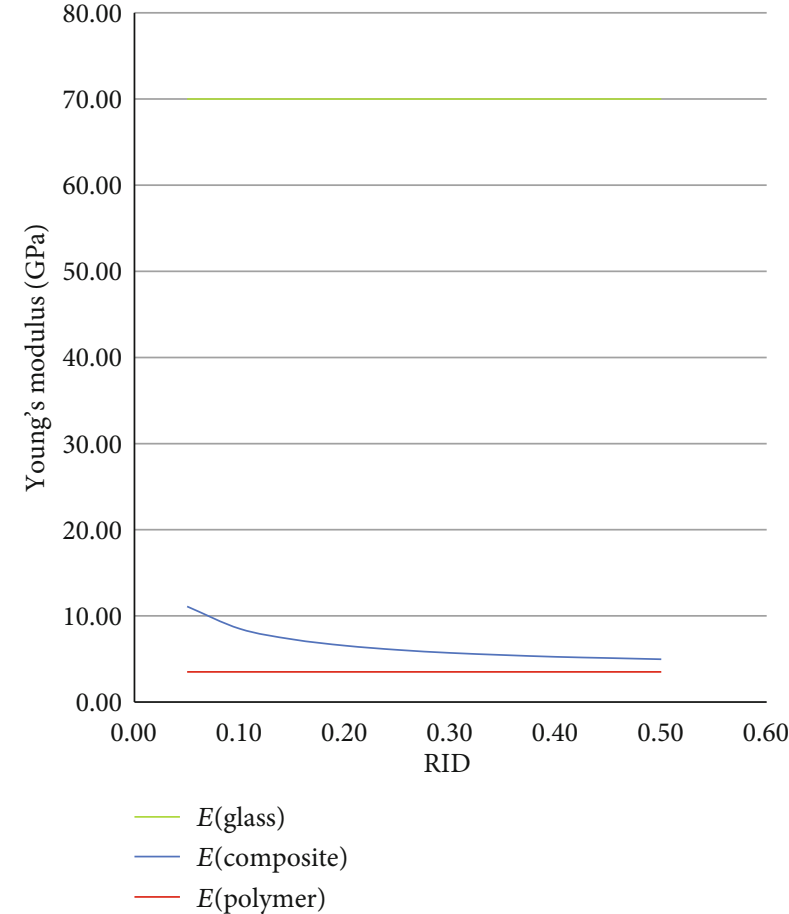

(b)

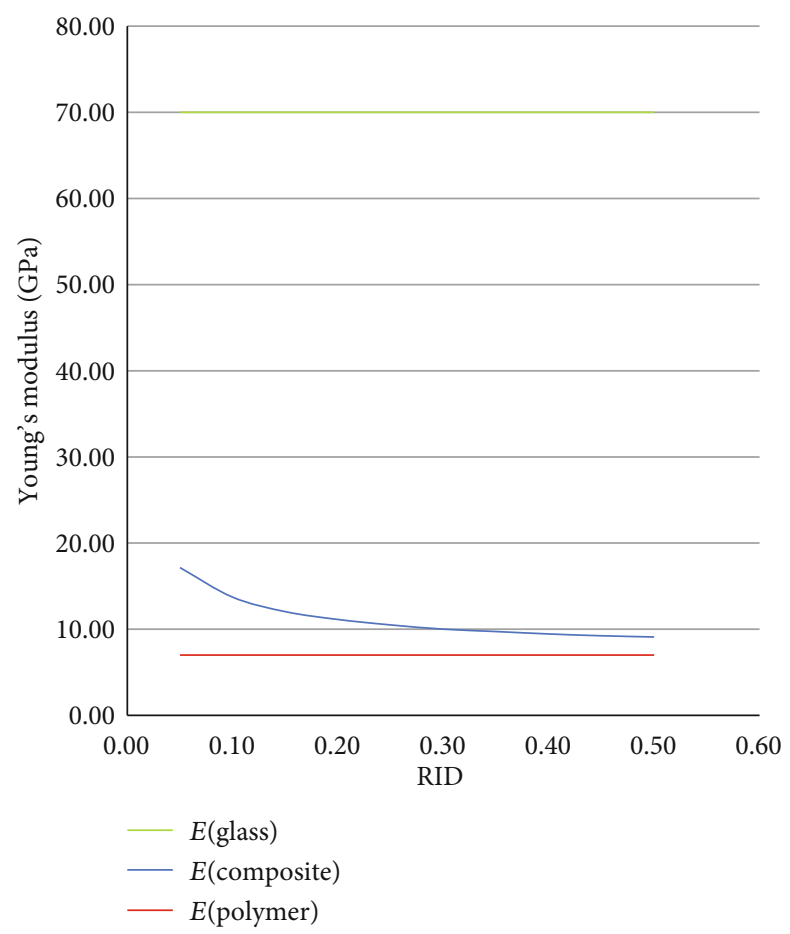

(c)

FIGURE 4: $E$ (composite) changed with RID when $E$ (substrate) was (a) $1.4 \mathrm{GPa}$, (b) $3.5 \mathrm{GPa}$, and (c) $7 \mathrm{GPa}$, respectively.

\section{Results}

By observing the data from FE modelling, it is clear that the indentation response will be affected by penetration and materials' properties. To investigate the effects from the penetration, Poisson's ratios of the coating and the substrate were initially fixed to 0.3 . Thereafter, their Poisson's ratios were changed to different values to differentiate how Poisson's ratio will affect the indentation response.

3.1. Glass $(v=0.3)$ on Different Polymers $(v=0.3)$

3.1.1. Young's Modulus of the Composite. In the simulation, 9 polymer combinations between the coating and the substrate 


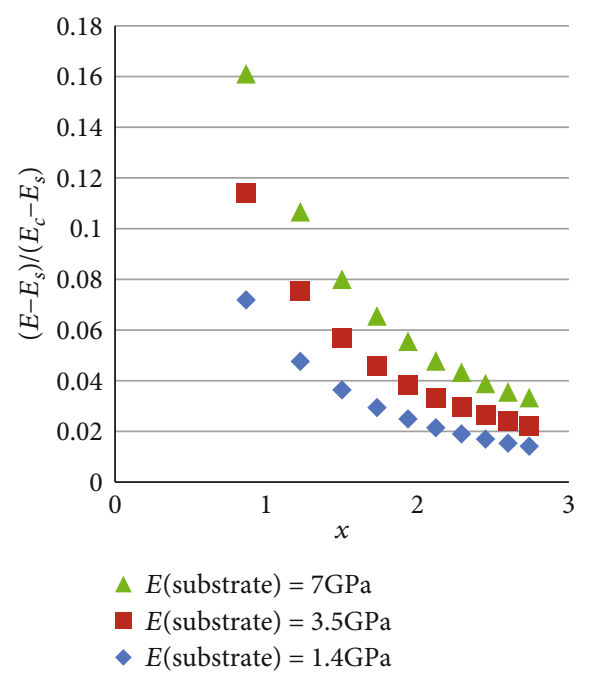

Figure 5: Effects of varying $E$ (substrate) on the mechanical response of the coating/substrate system.

were modelled, by fixing $E$ (coating) to $70 \mathrm{GPa}$, while varying $E$ (substrate) within $0.7 / 3.5 / 7 \mathrm{GPa}$ and viscoelasticity (i.e., $g_{1}$ ) within $0.1 / 0.5 / 0.9$. Higher viscoelasticity means more time for the system to reach the equilibrium, but it will not affect the final status. As for the substrate's Young's modulus, it has a positive relationship with the equilibrium loading force, which increases with the increasing penetration.

After the force reaches equilibrium during the stress relaxation period, Equation (5) can be rewritten as

$$
P_{\infty}=\frac{4}{3\left(1-v^{2}\right)} \sqrt{R} \delta^{3 / 2}(t) E_{\infty} .
$$

In order to simplify the calculation, another polymer bulk is used as the control group, and then, the relation between force and equilibrium Young's modulus can be derived as

$$
\frac{P_{\infty}(\text { composite })}{P_{\infty}(\text { polymer })}=\frac{E_{\infty}(\text { composite })}{E_{\infty}(\text { polymer })} .
$$

By analysing the force-depth data of different polymer bulks, the final loading force increases with the increasing Young's modulus of polymer bulks and penetration, while the viscoelasticity does not obviously influence the equilibrium status of the coating/substrate system.

The relationships between the force ratio and the relative indentation depth (RID, i.e., ratio of indentation depth to coating thickness) are depicted in Figure 3. By extracting the data from the equilibrium status, it is indicated that the force ratio (i.e., $P_{\infty}$ (composite) $/ P_{\infty}$ (polymer)) during the equilibrium status was changed with the indentation depth. Therefore, by rearranging Equation (12), E(composite) is calculated and depicted in Figure 4. It demonstrates that, for a fixed RID value, the force ratio decreases with the increase of $E$ (polymer), while Young's modulus of the composite will increase with the increase of $E$ (polymer). This may due to the fact that the coating layer is relatively thin and that Young's

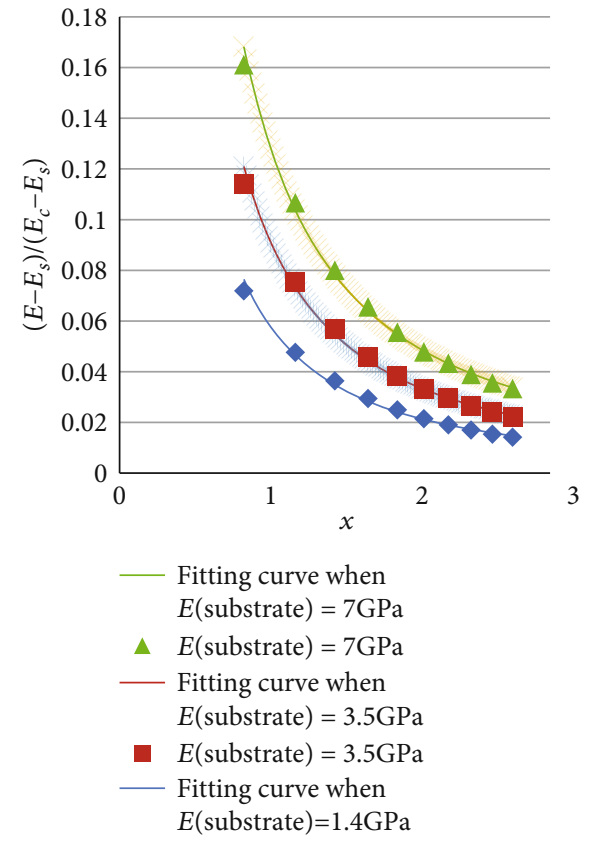

FIGURE 6: Fit $E$ (composite) by Equation (14), when $E$ (substrate) varies between $1.4 \mathrm{GPa}$ and $7 \mathrm{GPa}$.

TABLE 1: Results of the fitting parameters for Equation (14) found.

\begin{tabular}{lccc}
\hline$E$ (substrate) & $P$ & $Q$ & $n$ \\
\hline $7 \mathrm{GPa}$ & -6.98 & -0.17 & 0.9 \\
$3.5 \mathrm{GPa}$ & -9.25 & -0.19 & 0.9 \\
$1.4 \mathrm{GPa}$ & -14.13 & -0.19 & 0.9 \\
\hline
\end{tabular}

modulus of the composite rapidly transfers from coatingdominated to substrate-dominated, especially when RID reaches 0.1 .

3.1.2. Curve Fitting. In order to distinguish how the substrate affects the indentation responses, another figure about the relation between $\left(E^{*}-E_{\mathrm{s}}^{*}\right) /\left(E_{\mathrm{c}}^{*}-E_{\mathrm{s}}^{*}\right)$ and $\sqrt{R \delta} / h_{\text {thickness }}$ is needed. As shown in Figure 5, $\left(E^{*}-E_{\mathrm{s}}^{*}\right) /\left(E_{\mathrm{c}}^{*}-E_{\mathrm{s}}^{*}\right)$ decreases with the increasing $\sqrt{R \delta} / h_{\text {thickness }}$ and increases with the rising substrate's Young's modulus.

As $v$ (coating) is equal to $v$ (substrate), so Equation (9) can be derived as

$$
\frac{E^{*}-E_{\mathrm{s}}^{*}}{E_{\mathrm{c}}^{*}-E_{\mathrm{s}}^{*}}=\frac{E-E_{\mathrm{s}}}{E_{\mathrm{c}}-E_{\mathrm{s}}}
$$

where $E$ is Young's modulus of the composite, $E_{\mathrm{s}}$ is Young's modulus of the substrate, and $E_{\mathrm{c}}$ is Young's modulus of the coating. Then, Equation (10) can be rewritten as

$$
\frac{E-E_{\mathrm{s}}}{E_{\mathrm{c}}-E_{\mathrm{s}}}=\frac{P x^{n}}{1+P x^{n}+Q x}+1 \text {. }
$$




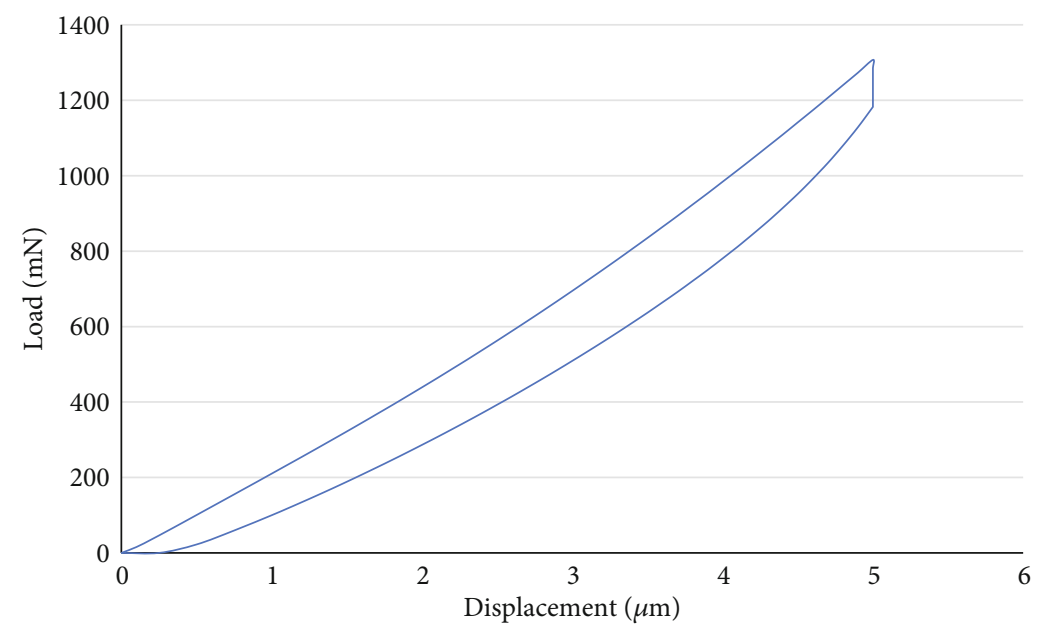

Figure 7: Schematic of the load-displacement curve for the coating/substrate system during the nanoindentation.

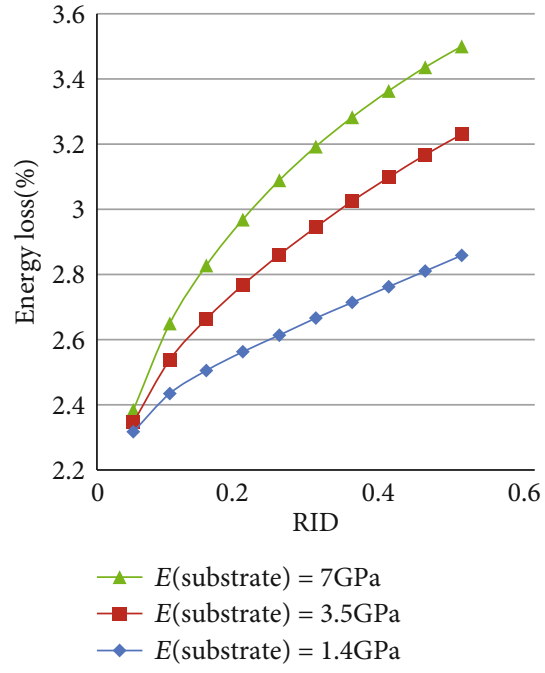

(a)

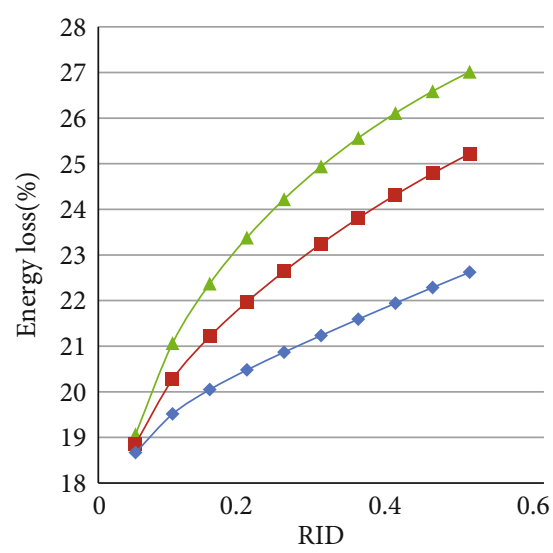

$$
\begin{aligned}
\multimap & E(\text { substrate })=7 \mathrm{GPa} \\
-E(\text { substrate }) & =3.5 \mathrm{GPa} \\
\rightarrow E(\text { substrate }) & =1.4 \mathrm{GPa}
\end{aligned}
$$

(b)

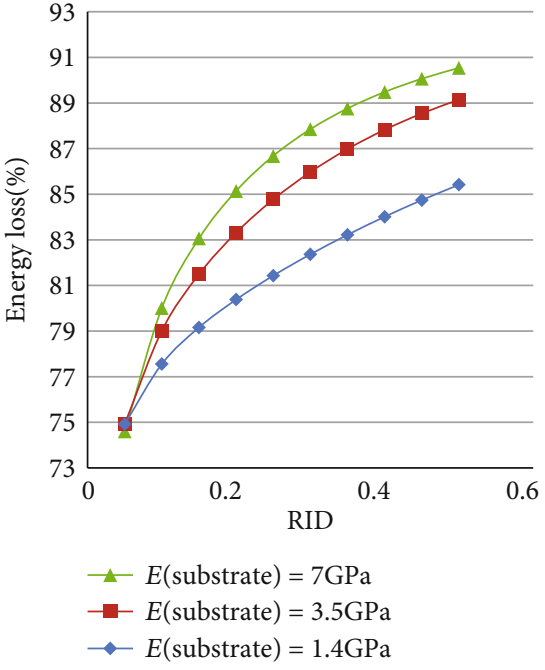

(c)

Figure 8: Energy loss of the coating/substrate system with $g_{i}$ (substrate) equal to (a) 0.1 , (b) 0.5 , and (c) 0.9 .

By fitting those data with Equation (14), curves are worked out with the fitting parameters of $P, Q$, and $n$, which have been shown in Figure 6 and Table 1, respectively.

The results suggest that, with the increase of $E$ (substrate), $P$ will be increasing, $Q$ may slightly change, but $n$ will not be affected. Compared with Figure 6, it shows that $P$ will affect the vertical location of the curve.

3.1.3. Energy Loss. Energy loss is the proportion of the difference between the total work and the recovery work to the total work. A schematic of such a graph is shown in Figure 7, the area underneath the loading curve is denoted as the total work, and the area underneath the unloading curve is denoted as the recovery work. Figure 8 demonstrates the energy loss of the glass on varying substrate materials during the nanoindentation.It shows a positive relationship between the energy loss and the penetration. Besides, Young's modulus of the substrate and the viscoelasticity of the substrate will also affect the energy loss of the coating/substrate system during the nanoindentation. The increasing Young's modulus of the substrate increases the energy loss; however, this increase is not obvious when penetration is not big enough. As for the viscoelasticity of the substrate, it makes a great contribution to the energy loss, as the energy loss is less than $3.6 \%$ when substrate's viscoelasticity equalled 0.1 but bigger than $73 \%$ when substrate's viscoelasticity increased to 0.9 .

3.2. Glass $(v=0.25)$ on Different Polymers $(v=0.5)$. Then, $v$ (glass) is changed to 0.25 and $v$ (polymer) is changed to 0.5 to differentiate how Poisson's ratio will affect the indentation response.

3.2.1. Young's Modulus of the Composite. The forcedisplacement curves indicated that force decreases rapidly during the stress relaxation period and reaches the equilibrium 


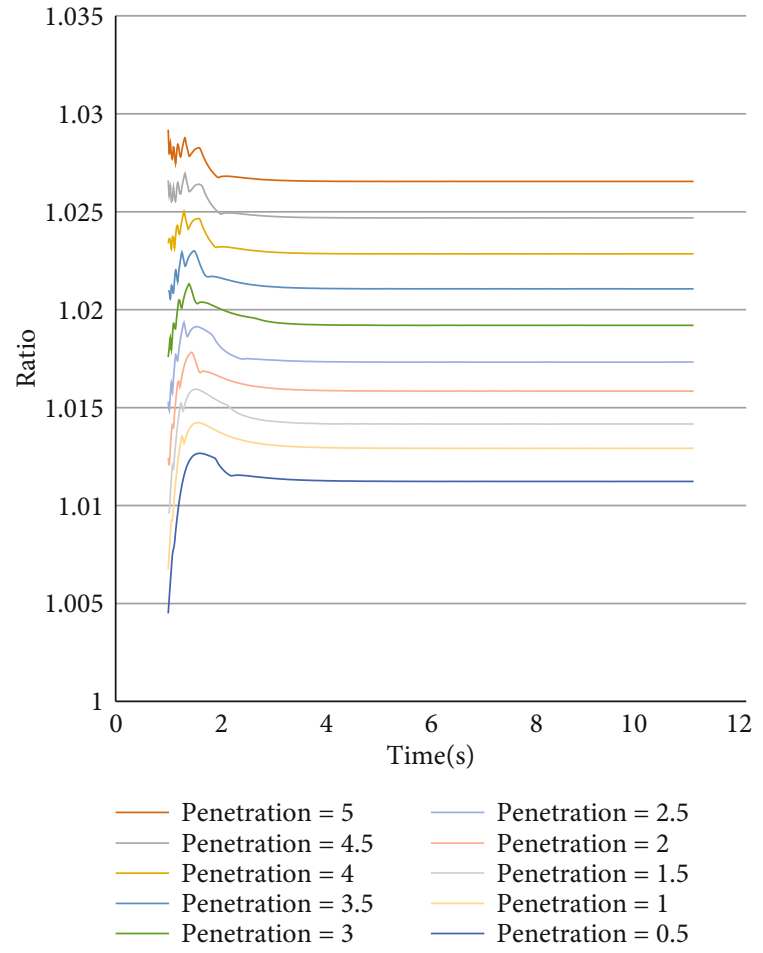

Figure 9: Ratios of force (where $v$ (substrate) equals 0.5 ) to force (where $v$ (substrate) equals 0.25 ) as a function of displacement, when $E$ and $g_{i}$ of the substrate equals $1.4 \mathrm{GPa}$ and 0.9 , respectively.

in the end. The increase of the viscoelasticity means more time for the coating/substrate system to reach the equilibrium; however, the change of viscoelasticity will not affect the final status (loading force when it reaches the equilibrium). On the other hand, the final force increases with the rising penetration and $E$ (substrate).

As $v$ (glass) and $v$ (polymer) are not same, the results from Equation (12) may have some deviation. Hence, another simulation, which changes $v$ (polymer) from 0.5 to 0.25 , should be done to discuss if Poisson's ratio will make a big difference. Here, just take one combination as an example, in which case $E$ and $g_{i}$ of the substrate equal $1.4 \mathrm{GPa}$ and 0.9 , respectively. Figure 9 illustrates the ratios of force (where $v$ (substrate) equals 0.5 ) to force (where $v$ (substrate) equals 0.25 ) as a function of displacement. Although those ratios will be slightly affected by the displacement, the change is not obvious with the values approximately equal to 1, which means Equation (12) is valid. Then, as shown in Figure 10, the ratios of Young's modulus for the coating/substrate system with different Poisson's ratios are calculated based on Equation (12). Those ratios decrease with the increasing RID and $E$ (substrate).

Figure 11 shows $E$ (composite) as a function of RID calculated from Equation (12). It is indicated that the results are independent with polymer's viscoelasticity. Although Young's modulus of the glass is relatively high $(70 \mathrm{GPa})$, Young's modulus of the composite is close to Young's modulus of the polymer, and this tendency will be much more obvious when the penetration becomes deeper. It indicates

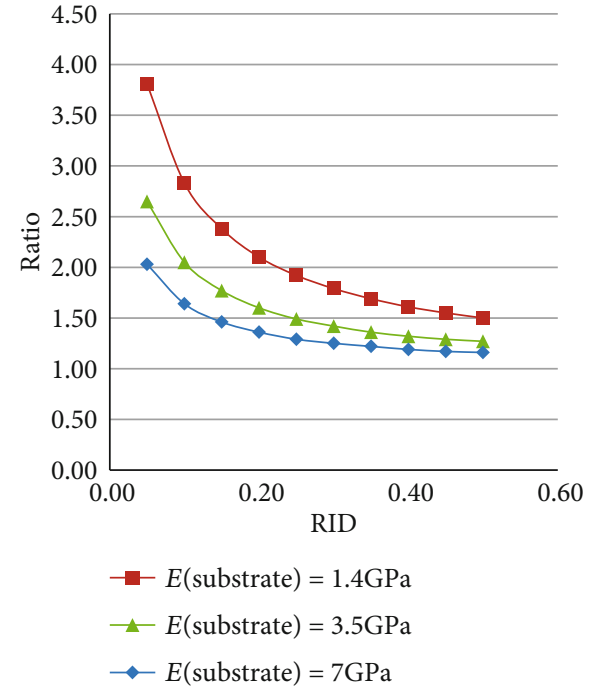

FIgURE 10: Ratios of Young's modulus for the coating/substrate system with $v$ (glass) equal to 0.25 and $v$ (polymer) equal to 0.5 , as a function of RID.

that Young's modulus of the composite is mainly substratedominated rather than coating-dominated, especially when RID reaches 0.1 .

3.2.2. Curve Fitting. In order to investigate the relationship between $E$ (composite) and penetration, Equation (10) is used. However, as $v$ (coating) and $v$ (substrate) are not equal, Equations (13) and (14) are invalid now, and Equation (10) is rewritten as

$$
\frac{\left(E /\left(1-v^{2}\right)\right)-\left(E_{\mathrm{s}} /\left(1-v_{\mathrm{s}}^{2}\right)\right)}{\left(E_{\mathrm{c}} /\left(1-v_{\mathrm{c}}^{2}\right)\right)-\left(E_{\mathrm{s}} /\left(1-v_{\mathrm{s}}^{2}\right)\right)}=\frac{P x^{n}}{1+P x^{n}+Q x}-1,
$$

where $v_{\mathrm{s}}=0.5$ and $v_{\mathrm{c}}=0.25$.

As Poisson's ratio of the composite is unknown, it assumes $v$ (composite) is equal to $0.3 / 0.38 / 0.45$ firstly. Hence, the fitting results are shown in Figure 12 and Table 2.

As shown in Table 2, when Poisson's ratio of the composite is equal to $0.3 / 0.38 / 0.45$, with the increase of $E$ (substrate), $n$ is similar to $1, Q$ is rising slightly, and $P$ will decrease. On the other hand, when $E$ (substrate) is fixed, with the rise of the composite's Poisson's ratio, $n$ is almost not affected. But the numbers of $P$ and $Q$ are quite similar, respectively, though they will increase in a slight rate.

3.2.3. Energy Loss. By calculating the total work and recovery work from each coating/substrate system, the energy loss of each system is computed and demonstrated in Figure 13. These curves shown in Figure 13 are almost identical to those curves shown in Figure 8, which indicates Poisson's ratio of the composite may not influence the energy loss.

\section{Discussion}

4.1. Young's Modulus of the Composite. As shown in Figures 3 and 4, fixed RID, when Young's modulus of the substrate 


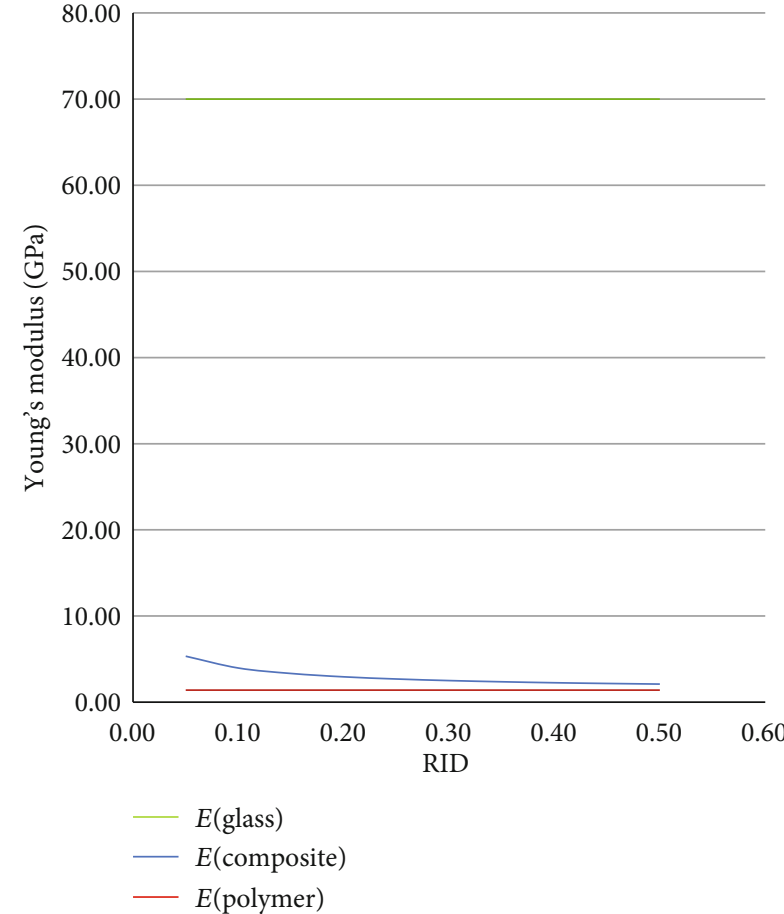

(a)

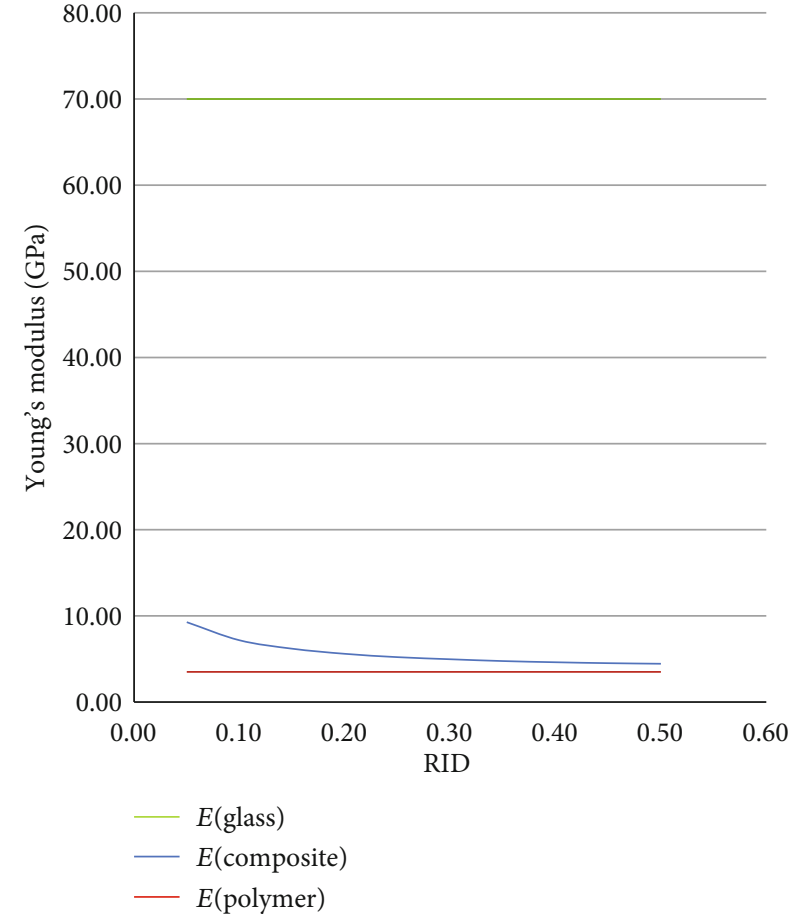

(b)

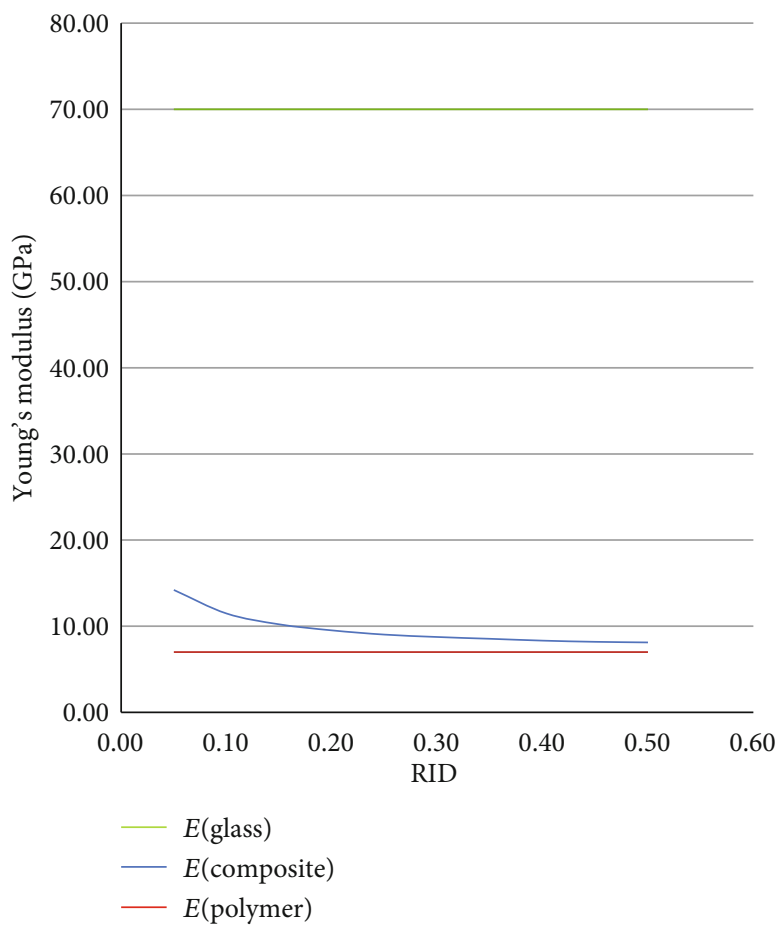

(c)

FIgURE 11: $E$ (composite) changed with RID when $E$ (substrate) is equal to (a) $1.4 \mathrm{GPa}$, (b) $3.5 \mathrm{GPa}$, and (c) $7 \mathrm{GPa}$.

increases from 1.4 GPa to $7 \mathrm{GPa}$, Young's modulus ratio will rapidly decrease to 1 and Young's modulus of the composite will be close to Young's modulus of the substrate when penetration rises from $0.5 \mathrm{~mm}$ to $5 \mathrm{~mm}$.

On the other hand, compared the data shown in Figures 4 and 11, Young's modulus of the composite decreases when Poisson's ratio of the composite increase from 0.3 to 0.38 .
With the increase of the penetration, E(substrate) or $v$ (composite), effects from the polymer are increasingly contributing to the indentation response.

4.2. Fitting Parameters. For the coating $(v=0.3) /$ substrate $(v=0.3)$ system, only $P$ increases with the increasing Young's modulus of the substrate, and $Q$ and $n$ are almost 


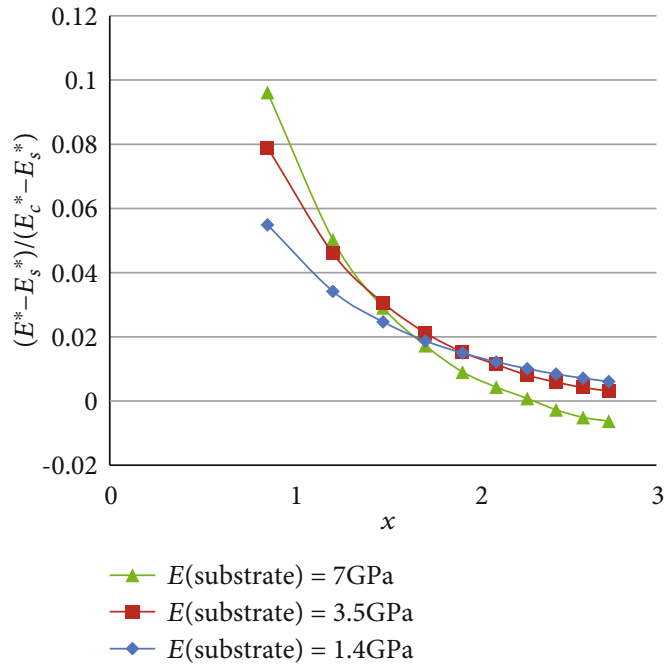

(a)

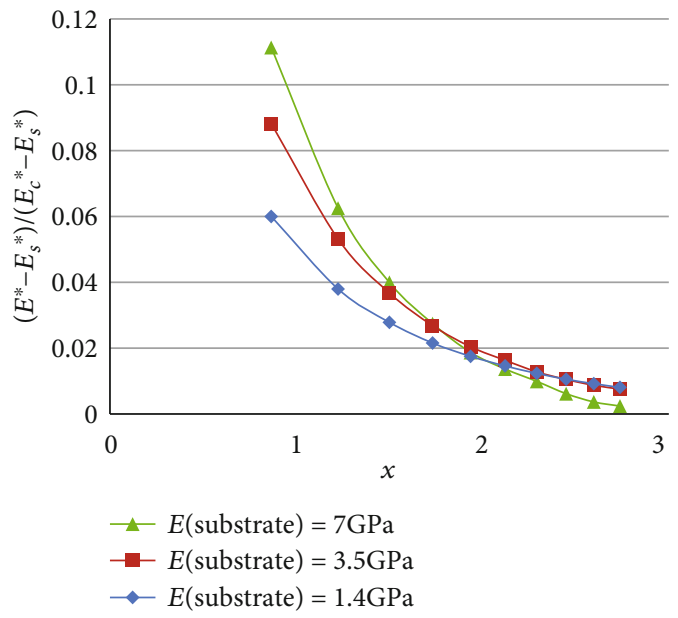

(b)

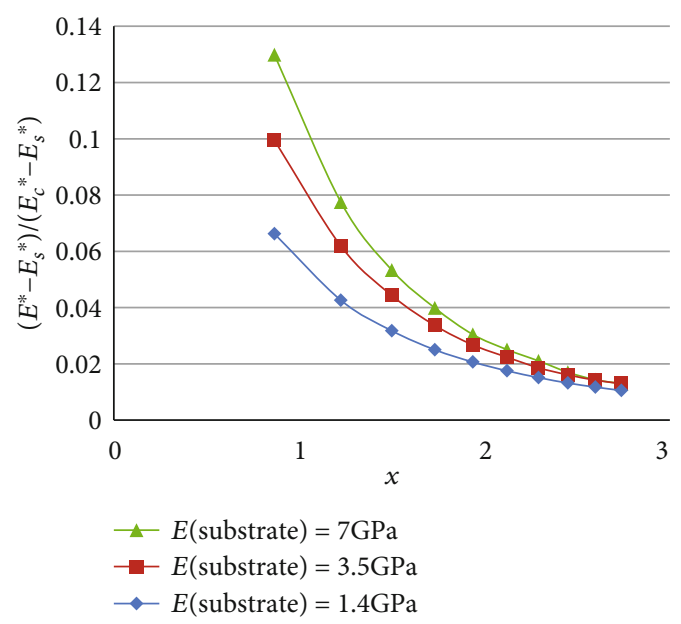

(c)

FIGURE 12: Fitting curves when $v$ (composite) equals (a) 0.3 , (b) 0.38 , and (c) 0.45 .

TABLE 2: Results of the fitting parameters.

\begin{tabular}{lcccc}
\hline$v$ (composite) & $E$ (substrate $)$ & $P$ & $Q$ & $n$ \\
\hline \multirow{3}{*}{0.3} & $7 \mathrm{GPa}$ & -16.77 & -0.27 & 0.95 \\
& $3.5 \mathrm{GPa}$ & -11.06 & -0.34 & 1 \\
& $1.4 \mathrm{GPa}$ & -8.27 & -0.43 & 1.04 \\
\hline \multirow{3}{*}{0.38} & $7 \mathrm{GPa}$ & -15.8 & -0.25 & 0.94 \\
& $3.5 \mathrm{GPa}$ & -10.53 & -0.3 & 1 \\
& $1.4 \mathrm{GPa}$ & -7.99 & -0.36 & 1.04 \\
\hline \multirow{3}{*}{0.45} & $7 \mathrm{GPa}$ & -14.81 & -0.22 & 0.94 \\
& $3.5 \mathrm{GPa}$ & -9.94 & -0.25 & 0.99 \\
& $1.4 \mathrm{GPa}$ & -7.66 & -0.27 & 1.03 \\
\hline
\end{tabular}

not affected. Hence, it seems that $P$ is determined by $E$ (substrate), when $v$ (coating) is equal to $v$ (substrate).

For the coating $(v=0.25) /$ substrate $(v=0.5)$ system, $P$ and $Q$ are influenced by both $E$ (substrate) and $v$ (composite). To be exact, $P$ decreases with the increasing Young's modulus of the substrate. And $n$ is just affected by $E$ (substrate), regardless the change of $v$ (composite).

4.3. Energy Loss. Comparing Figures 8 and 13, data from those different systems are almost the same. One possible explanation is that the composite's Poisson's ratio will not affect the energy loss of the system. Moreover, energy loss is mainly determined by $g_{i}$ (substrate), though it is also influenced by the penetration and substrate's Young's modulus. The energy loss will be higher than $95 \%$ when $g_{i}=0.9$ but less than $5 \%$ when $g_{i}=0.1$. On the other side, the change of the energy loss also indicates the trend and magnitude of the residual stress.

\section{Summary}

In this study, indentation responses of hard coating $(v=0.3)$ on 9 different substrates $(v=0.3)$ have been simulated and analysed firstly, and most of the work now focuses on the stress relaxation period. By calculating the ratio of Young's modulus, Young's modulus of the composite on each 


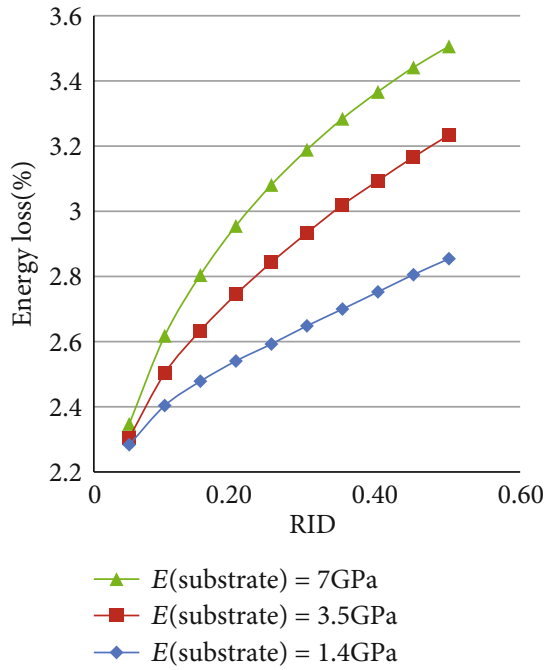

(a)

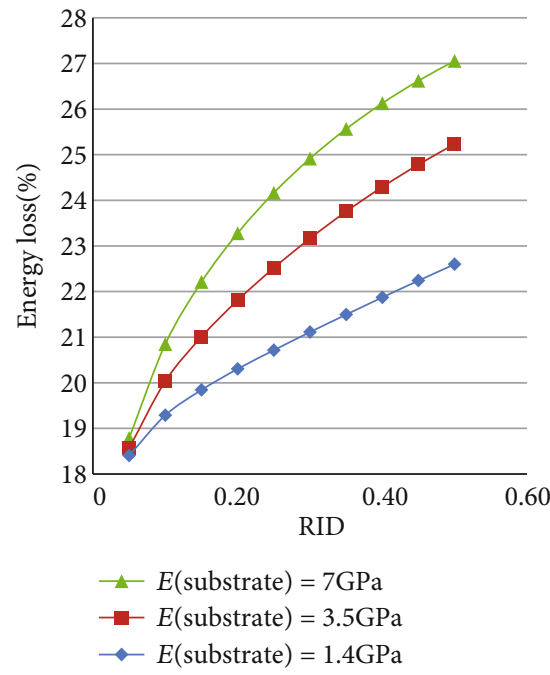

(b)

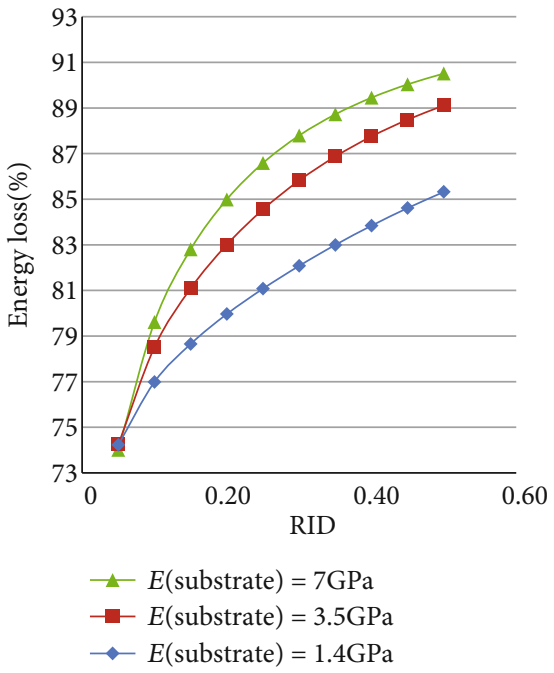

(c)

Figure 13: Energy loss of varying substrates when $g_{i}$ (substrate) equals (a) 0.1 , (b) 0.5 , and (c) 0.9 .

penetration depth has been obtained. Then, the relationships between $E$ (composite) and penetration on different substrates have been computed by fitting the curves.

Furthermore, data about energy loss have been demonstrated to investigate how it is affected by the substrate. By changing $v$ (coating) and $v$ (substrate) to 0.25 and 0.5 , respectively, the effects from Poisson's ratio have also been researched.

Till now, the effects from $E$ (substrate), $g_{i}$ (substrate), penetration, and Poisson's ratio have been discussed in the holding period, which can be used to predict the indentation response of the composite with different substrates. However, Poisson's ratio of the composite has not been worked out yet. Therefore, as a future work, more investigation will be needed on $v$ (composite) and the nanoindentation response in the ramping period.

\section{Data Availability}

The data used to support the findings of this study are available from the corresponding author upon request.

\section{Conflicts of Interest}

The authors declare that they have no conflict of interest.

\section{Acknowledgments}

The first author would like to thank Double Thousand Persons Plan for High-level Talents of Innovation and Entrepreneurship in Jiangxi Province and Scientific Research Innovation Team Support Project of Pingxiang University (201802). This work was financially supported by National Key R\&D Program of China (No. 2016YFC0209305) and Scientific Research Innovation Team Support Project of Pingxiang University (201802).

\section{References}

[1] Y. C. Lu, S. N. V. R. K. Kurapati, and F. Yang, "Finite element analysis of deep indentation by a spherical indenter," Journal of Materials Science, vol. 43, no. 18, pp. 6331-6336, 2008.

[2] D. R. Cairns, R. P. Witte II, D. K. Sparacin et al., "Straindependent electrical resistance of tin-doped indium oxide on polymer substrates," Applied Physics Letters, vol. 76, no. 11, pp. 1425-1427, 2000.

[3] H. Lee, S. M. Dellatore, W. M. Miller, and P. B. Messersmith, "Mussel-inspired surface chemistry for multifunctional coatings," Science, vol. 318, no. 5849, pp. 426-430, 2007.

[4] S. K. Hau, H.-L. Yip, N. S. Baek, J. Zou, K. O’Malley, and A. K.Y. Jen, "Air-stable inverted flexible polymer solar cells using zinc oxide nanoparticles as an electron selective layer," Applied Physics Letters, vol. 92, no. 25, article 253301, 2008.

[5] A. Pajares, L. Wei, B. R. Lawn, N. P. Padture, and C. C. Berndt, "Mechanical characterization of plasma sprayed ceramic coatings on metal substrates by contact testing," Materials Science \& Engineering A, vol. 208, no. 2, pp. 158-165, 1996.

[6] B. John, "Protective lithium ion conducting ceramic coating for lithium metal anodes and associate method," US Patent 5314765 A, 1994.

[7] J. Chen and S. J. Bull, "On the factors affecting the critical indenter penetration for measurement of coating hardness," Vacuum, vol. 83, no. 6, pp. 911-920, 2009.

[8] P. Duan and J. Chen, "Nanomechanical and microstructure analysis of extracellular matrix layer of immortalized cell line y201 from human mesenchymal stem cells," Surface \& Coatings Technology, vol. 284, no. 9, pp. 417-421, 2015.

[9] P. Duan, R. Toumpaniari, S. Partridge et al., "How cell culture conditions affect the microstructure and nanomechanical properties of extracellular matrix formed by immortalized human mesenchymal stem cells: an experimental and modelling study," Materials Science and Engineering: C, vol. 89, pp. 149-159, 2018.

[10] J. Chen and G. Lu, "Finite element modelling of nanoindentation based methods for mechanical properties of cells," Journal of Biomechanics, vol. 45, no. 16, pp. 2810-2816, 2012. 
[11] L. Min, C. Wei-Min, L. Nai-Gang, and W. Ling-Dong, "A numerical study of indentation using indenters of different geometry," Journal of Materials Research, vol. 19, no. 1, pp. 73-78, 2004.

[12] Y. Qin, Y. Li, L. Y. Zhang, and G. K. Xu, "Stochastic fluctuationinduced cell polarization on elastic substrates: a cytoskeletonbased mechanical model," Journal of the Mechanics and Physics of Solids, vol. 137, article 103872, 2020.

[13] G. K. Xu, B. Li, X. Q. Feng, and H. Gao, "A tensegrity model of cell reorientation on cyclically stretched substrates," Biophysical Journal, vol. 111, no. 7, pp. 1478-1486, 2016.

[14] G. K. Xu, X. Q. Feng, and H. Gao, "Orientations of cells on compliant substrates under biaxial stretches: a theoretical study," Biophysical Journal, vol. 114, no. 3, pp. 701-710, 2018.

[15] Y. Ding, G. K. Xu, and G. F. Wang, "On the determination of elastic moduli of cells by AFM based indentation," Scientific Reports, vol. 7, no. 1, 2017.

[16] Y. Ding, J. Wang, G. K. Xu, and G. F. Wang, "Are elastic moduli of biological cells depth dependent or not? Another explanation using a contact mechanics model with surface tension," Soft Matter, vol. 14, no. 36, pp. 7534-7541, 2018.

[17] I. Emri and N. W. Tschoegl, "Generating line spectra from experimental responses. Part I: relaxation modulus and creep compliance," Rheologica Acta, vol. 32, no. 3, pp. 311-322, 1993.

[18] A. Boccaccio, L. Lamberti, M. Papi et al., "A hybrid characterization framework to determine the visco-hyperelastic properties of a porcine zona pellucida," Interface Focus, vol. 4, no. 2, pp. 86-108, 2014.

[19] C. A. Clifford and M. P. Seah, "Nanoindentation measurement of young's modulus for compliant layers on stiffer substrates including the effect of poisson's ratios," Nanotechnology, vol. 20, no. 14, article 145708, 2009.

[20] M. L. Oyen, "Mechanical characterisation of hydrogel materials," International Materials Reviews, vol. 59, no. 1, pp. 4459, 2014.

[21] M. P. E. Wenger, L. Bozec, M. A. Horton, and P. Mesquida, "Mechanical properties of collagen fibrils," Biophysical Journal, vol. 93, no. 4, pp. 1255-1263, 2007. 